\title{
Process Management of Analgesia and Sedation Can Reduce the Incidence of Delirium
}

\author{
Hao Wang1, Shaolong Yi1, Hu Wang1, Minying Chen ${ }^{2 *}$ \\ ${ }^{1}$ Department of the Second Intensive Care Unit, The First People's Hospital of Kashi District, Kashi, China \\ ${ }^{2}$ Department of Surgical Intensive Care Unit, The First Affiliated Hospital of Sun Yat-sen University, Guangzhou, China \\ Email: *chmy1969@hotmail.com
}

How to cite this paper: Wang, H., Yi, S.L. Wang, H. and Chen, M.Y. (2019) Process Management of Analgesia and Sedation Can Reduce the Incidence of Delirium. Surgical Science, 10, 405-411. https://doi.org/10.4236/ss.2019.1011045

Received: November 11, 2019

Accepted: November 23, 2019

Published: November 26, 2019

Copyright (c) 2019 by author(s) and Scientific Research Publishing Inc. This work is licensed under the Creative Commons Attribution International License (CC BY 4.0).

http://creativecommons.org/licenses/by/4.0/

\section{cc) (i) Open Access}

\begin{abstract}
Background: Critical patients in ICU have to experience pain, anxiety, and sleep deprivation which always cause delirium, which will prolong the hospital stay and come up with higher mortality. Analgesia based sedation can reduce the accumulation of sedative effects, and shorten ventilator time and ICU length of stay. Process management of analgesia and sedation can reduce the incidence of delirium. Objectives: To explore the clinical benefits of procedural analgesia and sedation for critical ill patients. Methods: This is a prospective, two-phase study that focuses on patients who required mechanical ventilation after surgery. Comparing patients' pain and agitation scores, the species and dosage of sedative and analgesic, the incidence of delirium in the observation period and intervention period, data in two groups were collected and analyzed. Results: During the observational and interventional periods, we enrolled 213 patients before protocol implantation and 196 patients after protocol implantation. We found that there existed impropriate pain and sedation assessment in patients involved, and after training for procedural protocol, the average dosage of sedatives was decreased $(p>0.05)$. The percentage of reaching standard COPT score was $73.7 \%$ vs $84.1 \%(p>0.05)$ and RASS score was $70.9 \%$ vs $79.6 \%(p>0.05)$ in the observation period and intervention period, and the incidence of delirium was significantly reduced ( $31.9 \%$ vs $23.5 \%, p<0.05)$. Conclusion: We concluded that protocol implantation of analgesia and sedation can reduce the incidence of delirium.
\end{abstract}

\section{Keywords}

Procedural, Analgesia, Sedation, Intensive Care Unit, Delirium

\section{Introduction}

Critical patients in ICU have to experience pain, anxiety, and sleep deprivation 
which always cause mental disorder, namely delirium. Delirium is defined as an acute disturbance in attention and awareness with additional disturbances in cognition, with the exception of a pre-existing neurocognitive disorder [1], and is the most common mental complication in critically ill patients and associated with increased length of stay and long-term cognitive impairment. Chronic pain, sleep deprivation, and long hospital stay in ICU always put patients into high risk of delirium. Thus, the alleviation of pain and anxiety is an important component of ICU treatment strategies. The goals of analgesia and sedation are to minimize physical discomfort and pain, control anxiety, thus reducing the stress response and catabolism and reducing delirium [2]. The ideal state of patient under analgesia and sedation is comfortable, cooperative and calm. Insufficient analgesia and sedation can cause unexpected complications such as unplanned extubations [3] and exceeded analgesia and sedation can cause prolonged ventilation [4]. Delirium is also related to inappropriate analgesia and sedation. Currently accepted protocol of analgesic and sedation is analgesia first, with minimized sedation to avoid unexpected deep sedation [5]. Due to the close connection between delirium, agitation and pain, finding the best strategy of analgesia and sedation is urgent.

In this study, we first investigated the implementation of analgesia and sedation in ICU patient without intervention, and then compare the incidence of delirium after training medical staff for a standard analgesia and sedation procedure, aiming to find an appropriate strategy for analgesia and sedation in ICU patient and reduce the appearance of delirium.

\section{Data and Methods}

\subsection{Patients}

The inclusion criteria including the following: older than 18 years, need mechanical ventilation and agree to participate in this study. Exclusion criteria included the following: neurologic diseases, with liver or renal failure, drug abuse, pregnant, any situation not suitable for analgesia and/or sedation, patients who need deep sedation, and short-term expected mortality.

\subsection{Methods}

\subsubsection{Study Design}

The study was a prospective, two-phase (before-after), non-randomized and involved in patients who required mechanical ventilation after surgery. The first phase of this study was a two-month observational phase followed by data analysis in which none standard analgesia and sedation protocol was designed, and the level of pain and agitation, and whether needs to adjust medication was evaluated according to the experiences of the nurses. And then a two-month of interventional phase was applied after nurse and medical training for a fixed process of medication adjustment and pain and agitation assessment. Data in two groups were collected and analyzed. 


\subsubsection{Procedural Analgesia and Sedation}

The second phase of analgesia and sedation protocol was as following: the basic analgesia and sedation score was proceeded immediately on patients' admission to ICU, and then started medication with minimum dose. Analgesia and sedation score were played every $30 \mathrm{~min}$ for the first hour, and adjusted medication according to the score to achieve the target score, then do the assessment every $30 \mathrm{~min}$ after medication adjustment. If target score was achieved continuously twice, then do the assessment every $2 \mathrm{~h}$, and if target score was achieved continuously twice after, do the assessment every $4 \mathrm{~h}$, and do the assessment every $6 \mathrm{~h}$ if target score was achieved continuously twice until the study was over.

\subsubsection{Data Collection and Assessment Tools}

The following data in the two groups were collected: patient baseline, the type and dosage of analgesics and sedatives administered, analgesia and sedation score, the occurrence of delirium. The analgesic score used was Critical care pain Observation tool (CPOT score), and the sedative score used was Richmond Agitation-Sedation Scale (RASS score). The target analgesia score was CPOT $<3$, and target sedation score was $-2-1$. CAM-ICU score was used for delirium assessment every day.

\subsubsection{Outcome Measures}

The primary outcome was the incidence of delirium. The second outcome were the percentage of reaching analgesic and sedative target score, the species and dosage of sedative and analgesic in different periods.

\subsubsection{Statistical Analysis}

All numerical data are expressed as the mean \pm standard deviation. Student's $t$ test was used to compare the mean between the two groups, and chi-square test was used to compare the incidence between the two groups. $p<0.05$ was considered to indicate a statistically significant difference. SPSS 22.0 software (IBM SPSS, Armonk, NY, USA) was used to do statistical analysis.

\section{Results}

\subsection{Characteristics}

During the observational and interventional periods, we enrolled 213 patients in the observational group and 196 patients in the interventional group. The baseline characters of patients in two groups had no statistical difference (Table 1).

\subsection{Different Types of Analgesic and Sedatives between the Two Periods}

Fentanyl and Sufentanil were the most frequently used drugs for analgesia and sedation. Other medication included propofol, midazolam, Remifentanil, morphine. The type of drugs used in two phases had no statistical difference (Table 2). 
Table 1. Baseline characters of patients.

\begin{tabular}{cccc}
\hline Variables & Before intervention & After intervention & $p$ value \\
\hline Male & 119 & 113 & $>0.05$ \\
Age & $66 \pm 21$ & $64 \pm 19$ & $>0.05$ \\
APACHE II & $17 \pm 5$ & $19 \pm 3$ & $>0.05$ \\
\hline Operation type & & & \\
Trauma & 132 & 126 & $>0.05$ \\
Vascular surgery & 13 & 8 & $>0.05$ \\
Abdominal surgery & 27 & 23 & $>0.05$ \\
Other surgery & 41 & 39 & $>0.05$ \\
\hline
\end{tabular}

Table 2. Type of medications in different periods.

\begin{tabular}{cccc}
\hline Variables & Before intervention & After intervention & $p$ value \\
\hline Analgesic & & & \\
\hline Fentanyl & 132 & 118 & $>0.05$ \\
Sufentanil & 56 & 54 & $>0.05$ \\
Remifentanil & 25 & 24 & $>0.05$ \\
\hline Sedatives & & & $>0.05$ \\
\hline Dexmedetomidine & 104 & 116 & $>0.05$ \\
Propofol & 50 & 39 & $>0.05$ \\
Midazolam & 59 & 41 & \\
\hline
\end{tabular}

\subsection{Different Doses of Analgesic and Sedatives between the Two Periods}

The assessment of pain and sedation were proceeded $2.9 \pm 3.3$ times per day in observational patients and $7.6 \pm 2.1$ times per day in intervention patients. We compared the average rate and average daily dose of dexmedetomidine and remifentanil, and even though no statistical significantly, trend of less dose of dexmedetomidine was observed in the intervention group $(p>0.05)$ (Table 3$)$.

\subsection{Rate of Reaching Analgesic and Sedative Target and Incident of Delirium}

Before intervention, the percentage of achieved COPT score was 73.7\% (157/213) and RASS score was 70.9\% (151/213). After the procedural implantation, the percentage of achieved COPT score was $84.1 \%(165 / 196)$ and RASS score was 79.6\% (156/196), $p>0.05$. Meanwhile, 68 patients (31.9\%) presented delirium before intervention, as 46 patients $(23.5 \%)$ presented delirium after intervention $(p<0.05)$, significantly (Table 4$)$.

In summary, after the implantation of standardized analgesia and sedation procedures, the types of drugs used for critical ill patients did not change, and the drug dosage showed a little decreased, but there was no significant difference. The rate to reaching the target of analgesia and sedation had a tendency to 
Table 3. Dose of main medication in different periods.

\begin{tabular}{cccc}
\hline Variables & Before intervention & After intervention & $p$ value \\
\hline Dexmedetomidine $(\mu \mathrm{g} / \mathrm{kg} / \mathrm{h})$ & $0.65 \pm 0.16$ & $0.57 \pm 0.17$ & $>0.05$ \\
Fentanyl $(\mathrm{ug} / \mathrm{h})$ & $52 \pm 1.4$ & $46 \pm 1.7$ & $>0.05$ \\
\hline
\end{tabular}

Table 4. The percentage of analgesia and sedation target score and incident of delirium in different periods.

\begin{tabular}{cccc}
\hline Variables & Before intervention & After intervention & $p$ value \\
\hline CPOT score & $73.7 \%(157 / 213)$ & $84.1 \%(165 / 196)$ & $>0.05$ \\
RASS score & $70.9 \%(151 / 213)$ & $79.6 \%(156 / 196)$ & $>0.05$ \\
Delirium & $31.9 \%(68 / 213)$ & $23.5 \%(46 / 196)$ & $<0.05$ \\
\hline
\end{tabular}

increase, without significant difference. But the incidence of delirium was significantly reduced.

\section{Discussion}

In this two-phase study we found that procedural analgesia and sedation protocol can reduce delirium and optimize the effect of analgesia and sedative. In the observation phase, there assessment of pain and sedation was done almost every $8 \mathrm{~h}$ per day on average, and quite a few patients did not acquire appropriate pain relief or sedation. After training of procedural protocol, the assessment increased to nearly 8 times per day and more than $80 \%$ of patients acquire suitable analgesia and sedation. Meanwhile, the incidence of delirium was also decrease significantly following procedural protocol.

Nearly $40 \%$ percent of patients aged 65 or more developed into delirium during ICU stay [6]. Patients with delirium will have cognitive dysfunction and prolonged hospital stay, and also come up with higher mortality [7]. Risk factors of delirium including age, infection, length of hospital stay, sleep deprivation, the use of opioids and benzodiazepines [8]. Given these reasons, guidelines have recommended strategies for preventing and treating pain, agitation, and delirium [8].

Analgosedation was defined as analgesia-first sedation to make treatment pain a priority in providing sedatives, aiming to achieve and maintain a mild level of sedation [9]. Primary studies have demonstrated that analgosedation can reduce the accumulation of sedative effects, shortened ventilator time and ICU length of stay [10]. Use of non-benzodiazepine sedatives was approved to be associated with decreased incidence of delirium [11], and proper pain management, through routine assessment and monitoring of pain scale, improves the prognosis of patients treated in the ICU [8].

Present guidelines do not give recommendations about the frequency to do the assessment, and medical staff can only do this according to their experiences. High frequency of assessment may improve the effect of pain relief and the inci- 
dence of delirium, but it also brings some problem, such as increase the workload of nurses, and is difficult to achieve especially in the area where there exists shortage of medical staff. In addition to this, the frequency of assessment should base on the characteristics of the medications (i.e. long term or short term, the elimination half life). And how to adjust the dosage of the drug according to the present assessment score is also a great challenge.

Even if the negative outcome between classical and novel ant-delirium medications in delirium prevention which were latest published in New England journal of medicine [12], our study proved that procedural analgesia and sedation can reduce the incidence of delirium, and provide a possible protocol for pain and sedation, and recommended that once analgesia and sedation was proceeded on a patient, a protocol for assessment and medication adjustment should be accomplished. The protocol should be individual according to the manpower, the type of drugs and the experiences of clinician and nurses.

\section{Conclusion}

Delirium has poor effect on the prognosis for critical ill patients. Drug intervention doesn't effectively reduce the incidence of delirium. Our study shows that procedural analgesia and sedation can reduce the incidence of delirium. The protocol for assessment and medication adjustment should be accomplished individually according to the manpower, the type of drugs and the experiences of clinicians and nurses.

\section{Financial Support and Sponsorship}

This work was supported by the grants from Guangdong Science and Technology Innovation Strategy Special Fund Project (2018YJ026).

\section{Conflicts of Interest}

There are no conflicts of interest.

\section{References}

[1] Zaal, I.J. and Slooter, A.J. (2014) Light Levels of Sedation and DSM-5 Criteria for Delirium. Intensive Care Medicine, 40, 300-301. https://doi.org/10.1007/s00134-013-3192-5

[2] Taskforce, D.A.S., Baron, R., Binder, A., Biniek, R., Braune, S., Buerkle, H., Dall, P., Demirakca, S., Eckardt, R., Eggers, V., et al. (2015) Evidence and Consensus Based Guideline for the Management of Delirium, Analgesia, and Sedation in Intensive Care Medicine. Revision 2015 (DAS-Guideline 2015)-Short Version. GMS German Medical Science, 13.

[3] Lucas da Silva, P.S. and de Carvalho, W.B. (2010) Unplanned Extubation in Pediatric Critically Ill Patients: A Systematic Review and Best Practice Recommendations. Pediatric Critical Care Medicine, 11, 287-294. https://doi.org/10.1097/PCC.0b013e3181b80951

[4] Randolph, A.G., Wypij, D., Venkataraman, S.T., Hanson, J.H., Gedeit, R.G., Meert, K.L., Luckett, P.M., Forbes, P., Lilley, M., Thompson, J., et al. (2002) Effect of Me- 
chanical Ventilator Weaning Protocols on Respiratory Outcomes in Infants and Children: A Randomized Controlled Trial. The Journal of the American Medical Association, 288, 2561-2568. https://doi.org/10.1001/jama.288.20.2561

[5] Vincent, J.L., Shehabi, Y., Walsh, T.S., Pandharipande, P.P., Ball, J.A., Spronk, P., Longrois, D., Strom, T., Conti, G., Funk, G.C., et al. (2016) Comfort and Patient-Centred Care without Excessive Sedation: The eCASH Concept. Intensive Care Medicine, 42, 962-971. https://doi.org/10.1007/s00134-016-4297-4

[6] McNicoll, L., Pisani, M.A., Zhang, Y., Ely, E.W., Siegel, M.D. and Inouye, S.K. (2003) Delirium in the Intensive Care Unit: Occurrence and Clinical Course in Older Patients. Journal of the American Geriatrics Society, 51, 591-598.

https://doi.org/10.1034/j.1600-0579.2003.00201.x

[7] Fong, T.G., Jones, R.N., Marcantonio, E.R., Tommet, D., Gross, A.L., Habtemariam, D., Schmitt, E., Yap, L. and Inouye, S.K. (2012) Adverse Outcomes after Hospitalization and Delirium in Persons with Alzheimer Disease. Annals of Internal Medicine, 156, 848-856. https://doi.org/10.7326/0003-4819-156-12-201206190-00005

[8] Barr, J., Fraser, G.L., Puntillo, K., Ely, E.W., Gelinas, C., Dasta, J.F., Davidson, J.E., Devlin, J.W., Kress, J.P., Joffe, A.M., et al. (2013) Clinical Practice Guidelines for the Management of Pain, Agitation, and Delirium in Adult Patients in the Intensive Care Unit. Critical Care Medicine, 41, 263-306. https://doi.org/10.1097/CCM.0b013e3182783b72

[9] Devlin, J.W., Skrobik, Y., Gelinas, C., Needham, D.M., Slooter, A.J.C., Pandharipande, P.P., Watson, P.L., Weinhouse, G.L., Nunnally, M.E., Rochwerg, B., et al. (2018) Executive Summary: Clinical Practice Guidelines for the Prevention and Management of Pain, Agitation/Sedation, Delirium, Immobility, and Sleep Disruption in Adult Patients in the ICU. Critical Care Medicine, 46, 1532-1548. https://doi.org/10.1097/CCM.0000000000003298

[10] Karabinis, A., Mandragos, K., Stergiopoulos, S., Komnos, A., Soukup, J., Speelberg, B. and Kirkham, A.J. (2004) Safety and Efficacy of Analgesia-Based Sedation with Remifentanil versus Standard Hypnotic-Based Regimens in Intensive Care Unit Patients with Brain Injuries: A Randomised, Controlled Trial [ISRCTN50308308]. Critical Care Medicine, 8, R268-R280. https://doi.org/10.1186/cc2896

[11] Carson, S.S., Kress, J.P., Rodgers, J.E., Vinayak, A., Campbell-Bright, S., Levitt, J., Bourdet, S., Ivanova, A., Henderson, A.G., Pohlman, A., et al. (2006) A Randomized Trial of Intermittent Lorazepam versus Propofol with Daily Interruption in Mechanically Ventilated Patients. Critical Care Medicine, 34, 1326-1332. https://doi.org/10.1097/01.CCM.0000215513.63207.7F

[12] Girard, T.D., Exline, M.C., Carson, S.S., Hough, C.L., Rock, P., Gong, M.N., et al. (2018) Haloperidol and Ziprasidone for Treatment of Delirium in Critical Illness. The New England Journal of Medicine, 379, 2506-2516. https://doi.org/10.1056/NEJMoa1808217 